\title{
Cursos de Medicina com currículos inovadores são mais caros? \\ Análise de custos do Curso Integrado de Graduação em Medicina da Universidade Estadual de Londrina
}

PALAVRAS-CHAVE

- Análise de Custo.

- Custo do Ensino.

- Educação Médica.

\section{Are innovative medical curricula more costly? Cost analysis of the Medicine Course of the State University of Londrina}

Celita Salmaso Trelha Luis Fernando Casarim ${ }^{1}$ Marcio José de Almeida ${ }^{1}$ Pedro A. Gordan ${ }^{1}$

\section{R E S U M O}

O objetivo deste estudo foi analisar os custos do curso de graduação em Medicina da Universidade Estadual de Londrina. Foi realizado um estudo de caso, prospectivo, com levantamento das horas de dedicação dos professores às atividades acadêmicas e de gerenciamento do curso e número de docentes equivalentes a regime de tempo integral. No levantamento das informações utilizou-se o Cost Construction Model, e a coleta dos dados foi realizada por meio de análise documental e entrevistas. Esta metodologia permite separar custos instrucionais e custos compartilhados, de maneira a deixar claras eventuais diferenças de custo relacionadas ao desenho curricular. Foi realizada a identificação das despesas totais, alocação nas atividades-base, apoio e fins, e distribuição das despesas diretas e indiretas por meio do sistema de absorção. Participaram das atividades do curso 222,2 equivalentes a professores de regime de 40 horas. $O$ total de "horas contato" foi de 52.284. As atividades da primeira à quarta série implicaram um custo geral de $R \$$ $855.586,74$ e de $R \$ 1.629,025.93$ para os dois anos de internato. Incluindo-se o custo compartilhado equivalente ao curso de Medicina do hospital universitário, o custo total do curso foi estimado em $R \$ 15.643 .660,89$, e o custo aluno/ano foi de $R \$ 31.162,67$, o equivalente a US\$10,633.86. Este valor, apesar de cenários diversos e metodologias de apuração diferentes, está aquém dos custos estimados em universidades estrangeiras ou mesmo brasileiras.

\begin{abstract}
A B S T R A C T
The aim of this prospective case study was to conduct a cost analysis of the Integrated Problem-Based Medical Course of the Health Sciences Center of the State University of Londrina, Brazil, during the fiscal year 2005. This analysis was done in two phases. First, using a Cost Construction Model, the administrative and educational faculty contact hours during all educational activities were identified through analysis of documents and interviews with the teaching body and students. Second, all direct and indirect costs required for supporting the course were added through a Cost Absorption Method done by the Planning and Development Office of the University, which included all shared costs. In 2005 the medical course had 502 medical students enrolled and 284 teachers (222,2 full-time equivalents) were necessary to accomplish all educational and management tasks. For the first four years 18,932 and for the internships (years five and six) 33,352 faculty contact hours were needed. The hours allocated for administrative tasks amounted to 13.173. The estimated cost for the first four years was $R \$$ $855,586.74$ and for the internships $R \$ 1,629,025.93$. The administrative costs were $R \$ 586,642.21$. The share of the University Hospital was of $R \$ 12,522,406.00$ so that the total cost of the medical course was estimated in $R \$$ $15,643,660.89$, which corresponds to $R \$ 31,162.67$ or US $\$ 10,633.86$ (2005/dollar) per student/year. Due to the use of different approaches and diverse scenarios, cost comparisons with other institutions, local or international, are difficult but that could be overcome by using methods like the one used in this case study, where educational costs are established based on the design of the curriculum and separated from other administrative or shared costs.
\end{abstract}

Recebido em: 08/07/2007 Reencaminhado em: 21/12/2007 Aprovado em: 22/12/2007.

REVISTA BRASILEIRA DE EDUCACCÃO MÉDICA 


\section{INTRODUÇÃO}

O termo "custo", por vezes, é empregado de forma equivocada, representando para o indivíduo o sacrifício de recursos. Segundo Velloso ${ }^{1}$, custo é sinônimo de despesa. Diante desse conceito de custo como o sacrifício de recursos para obtenção de um objeto, no caso a educação superior, duas faces podem ser observadas: o custo para o aluno e o custo para a instituição. O custo para o estudante representa despesas relacionadas a aquisição de livros, fotocópias, taxas, transporte e mensalidades em escolas particulares, entre outros. Já o custo da instituição pública representa o "sacrifício" com que a sociedade arca para fornecer a educação superior ${ }^{2,3}$.

Sob o ponto de vista econômico, entende-se por custo toda e qualquer aplicação de recursos, para a produção e distribuição de mercadorias ou prestação de serviço ${ }^{4}$.

Megliorini ${ }^{5}$ define custos como "os gastos, não investimentos, necessários para fabricar os produtos da empresa. São os gastos efetuados pela empresa que farão nascer os seus produtos".

O tema custos alcança proporções consideráveis, principalmente no âmbito dos recursos públicos, elencando junto à sociedade brasileira maior confiança no Estado, seja na prestação de serviços ou na aquisição de produtos, os quais, se não forem analisados com eficácia, podem causar grandes déficits nos cofres públicos ${ }^{6}$.

As questões relativas a custos são de extrema importância na gestão econômica e estrutural das empresas, inclusive das que prestam serviços a seus clientes, como no caso das instituições de ensino. Na área da educação superior, a situação é precária, uma vez que estas atividades não possuíam um caráter empresarial que impulsionasse seus gestores ao estudo e à aplicação de técnicas eficazes no gerenciamento de recursos ${ }^{7}$.

A gestão de custos em qualquer tipo de organização é apresentada como um instrumento gerencial fundamental para o controle dos recursos, sejam eles financeiros, materiais ou patrimoniais. A determinação do custo pode estar ligada a diferentes aspectos: apuração e controle de gastos, fixação de preços, apuração de resultado, tomada de decisões, transparência e gestão de organização ${ }^{8}$.

Os estudos relacionados ao levantamento de custos nas escolas médicas nos Estados Unidos começaram no início da década de 1970, período em que o governo federal propiciou a expansão de novos cursos. Em 1974, a Associação de Escola de Medicina Americana publicou um estudo de custo de 12 escolas médicas. Os componentes analisados foram custos para instrução, pesquisa, atividades clínicas e profissionais, e atividades administrativas. O estudo obteve como resultado a média de US $\$ 19,800$, sendo US\$10,700 exclusivamente relacionados ao ensino 9 .
Rein et al. ${ }^{10}$ apuraram um custo anual para o aluno de Medicina na Universidade de Virgínia, no período de 1994-95, de US\$ 89,258 e levantaram a necessidade de 223 professores em tempo integral, sendo 7 professores das ciências básicas e 216 clínicos, para graduar 556 estudantes. Do custo total, US\$29,400 foram destinados ao pagamento de salários de professores e encargos, e US\$ 4,100 ao suporte ao programa de ensino e serviços administrativos.

Franzini et al. ${ }^{11}$ estimaram o custo do curso de Medicina na Universidade do Texas - Houston Medical School, no período de 1994-95, em US\$43,993 por estudante/ano para formar 800 estudantes de Medicina e a necessidade de 201 professores em regime integral, sendo 11 professores das ciências básicas e 190 clínicos.

O custo do ensino superior no País tem merecido atenção de pesquisadores e órgãos governamentais ${ }^{12,13}$. Na Lei Complementar no 101 / 2000, o art. 50 refere-se à manutenção de sistemas de custos para avaliação e acompanhamento da gestão orçamentária, financeira e patrimonial ${ }^{14}$. O Tribunal de Contas da União (TCU), por meio do Relatório Consolidado de Auditoria Operacional, recomendou a apuração do custo por aluno nas universidades federais, para avaliação de desempenho ${ }^{13}$.

No Brasil, a apuração de custos vem sendo mais amplamente realizada a partir da década de 1970. Em 1973, o MEC, em convênio com a Assessoria de Planejamento e Controle da UnB, divulgou estudo de custos e alocação de recursos, com base no modelo desenvolvido pelo National Center for Higher Education Management Systems do Colorado, EUA ${ }^{15}$. A Universidade Federal da Bahia (UFBA), também em 1973, apresentou um modelo de apropriação de custos para as universidades federais ${ }^{16}$.

Em 1994, o Ministério da Educação e do Desporto expediu o Sistema de Apuração de Custos das Instituições Federais de Ensino Superior, que orientava as Ifes a apurarem o custo. O sistema único para todas as instituições utilizava um software fornecido pelo MEC, e cada instituição definia a fonte de informações ${ }^{17}$.

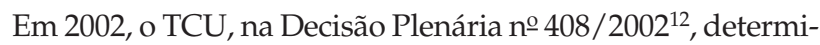
nou que as Ifes incluíssem no relatório de gestão anual os indicadores: custo corrente por aluno; relação aluno/professor; relação aluno/funcionário; relação funcionário/professor; grau de participação estudantil; grau de envolvimento com pós-graduação; conceito Capes; índice de qualificação do corpo docente; e taxa de sucesso da graduação. A decisão tinha o objetivo de elaborar um diagnóstico da educação superior em nível nacional e permitir a comparação entre as instituições.

Morgan ${ }^{18}$ determinou o custo por aluno na educação superior da Universidade de Brasília. Segundo a autora, o custo do ensino representa $29,06 \%$ das despesas orçamentárias e $59,97 \%$ do custo total das Unidades Acadêmicas. A Faculdade de Medicina apresentou o mai- 
or custo da universidade, estimado em $\mathrm{R} \$ 9.622 .842,00$. O custo por aluno apurado foi da ordem de $\mathrm{R} \$ 15.609,00$.

No ensino superior, o salário dos professores é o fator principal no custo da educação médica ${ }^{19,20}$. O elevado custo dos cursos de Medicina se relaciona principalmente ao salário do professor e à utilização de instalações hospitalares, equipamentos e laboratórios. Além disso, o curso de Medicina tem duração maior que outros cursos ${ }^{21}$. De acordo com Brown e Gamber ${ }^{22}$, cerca de $80 \%$ a $90 \%$ do custo são atribuídos a salários. A manutenção da biblioteca é apontada também como determinante substancial de custo.

A educação é uma das áreas em que os gastos têm crescido de forma rápida. Esse crescimento aumenta a necessidade de melhorar o gerenciamento da utilização dos recursos educacionais na gestão econômica e estrutural das instituições de ensino.

O curso de Medicina da Universidade Estadual de Londrina foi criado em 1965 pelo Decreto Estadual no 521, de 21 de dezembro de 1965, e iniciou suas atividades em 1967, com um currículo tradicional. Desde então, já promoveu cinco reformas curriculares ${ }^{23}$.

O projeto pedagógico vigente do curso está fundamentado na pedagogia interativa, com um eixo metodológico que confere destaque à Aprendizagem Baseada em Problemas e orientado para a comunidade. As atividades docente-assistenciais são centradas no estudante, visto como sujeito da aprendizagem, e no professor como facilitador. Apresenta ainda as seguintes diretrizes:

- Estruturação modular, que viabiliza a interdisciplinaridade; ensino centrado nas necessidades de aprendizagem dos estudantes.

- Currículo nuclear comum a todos os estudantes e a oportunidade de módulos e práticas eletivos, cuja função é permitir certa individualização

- Ensino baseado na pedagogia da interação, com os conteúdos das ciências básicas e clínicas desenvolvidos de forma integrada com os problemas prioritários de saúde da população.

- Garantia de contato do estudante de Medicina com as realidades de saúde e socioeconômicas da comunidade desde o primeiro ano do curso; adoção da avaliação formativa

- Terminalidade do curso em seis anos ${ }^{23}$.

O sistema acadêmico adotado pelo curso de Medicina é o seriado anual, com a oferta de três tipos de atividades: módulos temáticos (anuais ou em bloco), estágios supervisionados e módulos de atualização. As atividades acadêmicas são distribuídas em módulos temáticos interdisciplinares, desenvolvidos por meio das seguintes atividades pedagógicas: sessão tutorial; prática de laboratório e discussão de casos clínicos; palestras ou conferências; estudo orientado; avaliação; módulos de habilidades clínicas e atitudes; módulos de interação entre ensino, servi- ços de saúde e comunidade; módulos de atualização; estágios do internato ${ }^{23}$.

O objetivo deste estudo foi analisar o custo do curso de graduação em Medicina da Universidade Estadual de Londrina com currículo integrado no ano de 2005.

\section{MATERIAL E MÉTODO}

Para atingir o objetivo proposto, foi realizado um estudo de caso prospectivo. De acordo com Beuren ${ }^{24}$, o estudo de caso possibilita conhecer com maior profundidade um assunto ainda pouco explorado. Na área empresarial, o estudo de caso demonstra com profundidade a complexidade da contabilidade e do controle nas organizações internato ${ }^{25}$.

O levantamento do custo instrucional (custo diretamente relacionado à educação) baseou-se no Cost Construction Model e foi adaptado às características do programa educacional do curso de Medicina da UEL. O Cost Construction Model foi desenvolvido pela National Academy of Sciences e Association of American Medical Colleges e vem sendo utilizado para estimar o custo da educação médica de escolas de Medicina dos Estados Unidos e Canadá ${ }^{10,11,26,27}$.

Este modelo se baseia na análise do currículo a partir da descrição do programa, número de alunos, cronograma de atividades educacionais, carga horária docente destinada diretamente ao ensino e níveis salariais de docentes.

Foi efetuado um trabalho de campo para registrar as horas de dedicação dos professores envolvidos no curso de Medicina. Foram levantadas as cargas horárias dos docentes em contato com o aluno (horas contato) durante a graduação e as cargas horárias dos docentes envolvidas no gerenciamento do curso.

A coleta de dados ocorreu de fevereiro a dezembro de 2005, por meio de observação, análise documental e entrevistas. Os documentos analisados foram: manuais das atividades acadêmicas, atas de reuniões, resoluções e portarias. Essa análise documental possibilitou colher dados relativos principalmente à organização acadêmico-administrativa.

As entrevistas tiveram o intuito de levantar o número de horas contato, com os coordenadores das atividades acadêmicas, assistentes administrativos e representantes de alunos de todas as séries.

O presente estudo foi aprovado pela Comissão de Ética em Pesquisa da Universidade Estadual de Londrina. Participaram da pesquisa somente os entrevistados que assinaram o Termo de Consentimento Livre e Esclarecido.

Na seqüência, fez-se o levantamento do número equivalente de docentes em regime integral e a lotação por departamento. 
Foi considerado como referência o docente de tempo integral (40 horas semanais, com ou sem dedicação exclusiva), convertendo-se proporcionalmente os que se enquadram em outros regimes de dedicação.

No presente trabalho, todos os recursos que, direta ou indiretamente, foram aplicados nas diversas unidades administrativas e acadêmicas da instituição foram alocados aos Departamentos Acadêmicos, para, depois, serem apropriados aos programas, projetos e atividades efetivamente desenvolvidos no período.

Desse modo, os recursos diretamente aplicados nos Departamentos Acadêmicos constituem "despesas diretas" das atividades desenvolvidas. Considerando que o principal item de custo dos Departamentos Acadêmicos se refere à remuneração docente, será este o item de custo escolhido como vetor na definição da matriz de alocação aos respectivos departamentos, dos demais recursos aplicados pela instituição na realização de seus objetivos e na manutenção de sua máquina administrativa e acadêmica.

Uma vez que cada Departamento Acadêmico constitui um Centro de Custo, o estabelecimento dos fatores de alocação, para fins de rateio das despesas indiretas, terá como base o montante dos dispêndios incorridos com a remuneração docente por cada um dos Departamentos. Desse modo, se o montante da remuneração do corpo docente (despesa direta) do Departamento Acadêmico " $X$ " for equivalente a 10\% do total dos dispêndios dessa rubrica, este percentual será o fator utilizado para fins de alocação dos custos indiretos ao respectivo Departamento Acadêmico " $X$ ", e assim por diante.

Dessa forma, os Departamentos Acadêmicos acumularão as despesas diretas e indiretas, para depois apropriá-las aos programas e projetos executados e às atividades realizadas no período.

O sistema de custeio adotado neste trabalho e na UEL é o de custos por absorção, no qual se realiza a apropriação de algumas despesas com vistas a identificar, apurar e reunir os custos aplicados no desenvolvimento dos serviços, relacionando os fatos físicos com os monetários ${ }^{28}$. A apropriação tem o significado de atribuição e alocação.

A apropriação do custo do processo e de uma parcela do custo complementar é feita aos Centros de Custo. Após, através do custo-hora e do tempo aplicado nas diversas atividades, procede-se à apropriação destes custos aos serviços, juntamente com os demais itens de custos considerados. Os cálculos têm dois objetivos: identificar os custos de cada órgão e unidade (secundário) e os custos dos serviços (principal) ${ }^{28}$.

Todas as informações referentes aos custos foram provenientes da Pró-Reitoria de Planejamento (Proplan) da Universidade Estadual de Londrina.
A apuração do custo aluno de Medicina da UEL resultou da divisão dos custos apropriados ao curso pelo número de alunos. Foram considerados no cálculo todos os alunos matriculados no curso de graduação de Medicina no ano letivo de 2005.

Para o cálculo do custo hospital universitário no curso de Medicina, foi utilizado, e tido como válido, o critério adotado pela UEL, em que se realiza a diluição do custo do hospital na atividade de graduação proporcional ao peso do curso/aluno frente à atividade de estágio ${ }^{28}$.

O valor encontrado foi convertido em dólar americano pela média anual praticada no mercado financeiro no ano de 2005.

\section{RESULTADOS}

Em 2005, estavam matriculados no curso de Medicina da UEL 502 estudantes, distribuídos da primeira a sexta séries. Participaram das atividades acadêmicas 284 professores, sendo 84 $(29,6 \%)$ em regime integral de dedicação exclusiva; 68 (23,9\%) em 40 horas semanais; 4 (1,4\%) em 34 horas semanais; 28 (9,9\%) em 34 horas semanais; e 100 (35,2\%) em regime de 20 horas semanais - o equivalente a 222,2 professores em regime de 40 horas. Os docentes que desenvolveram atividades no curso de Medicina tinham vínculos de contrato temporários - 31 (10,9\%) - e efetivos - 253 (89,1\%). A formação dos docentes incluiu os níveis de: graduação, 20 (7,0\%); especialização, 48 (16,9\%); mestrado, 104 (36,6\%); doutorado, 106 (37,3\%); e pós-doutorado, 6 (2,2\%). Sete técnico-administrativos, lotados no Centro de Ciências da Saúde, desenvolveram atividades no curso.

Os professores do curso de Medicina da UEL desempenharam as seguintes atividades didáticas: tutor, palestrante ou conferencista, instrutor de atividades práticas, avaliador de atividades teóricas e práticas, instrutor de práticas de interação ensino, serviços e comunidade, instrutor de habilidades, avaliador de habilidades e supervisor de estágio de internato.

A hora em que o professor esteve em atividade em contato direto com o estudante foi considerada "hora contato docente". Da primeira à quarta série do curso de Medicina, foi levantado o total de $18.932,0$ horas contato, desempenhadas por 270 professores das Ciências Básicas e Clínicas.

Os professores envolvidos da primeira à quarta série do curso de Medicina eram provenientes dos Centros de Ciências Biológicas, Centro de Ciências Exatas, Centro de Letras e Ciências Humanas, Centro de Tecnologia e Urbanismo e, principalmente, do Centro de Ciências da Saúde. Dezenove Departamentos participaram do curso. O maior volume de horas contato docente foi do Departamento de Clínica Médica (6.118,5 horas) e Clínica Cirúrgica (3.226,5 horas), como consta na Tabela 1. 
TABELA 1

Distribuição das horas contato docente por série do curso de Medicina da UEL e Departamentos envolvidos. Londrina, 2005

\begin{tabular}{|c|c|c|c|c|c|}
\hline DEPARTAMENTOS/CENTROS & 1aㅗ Série & 2a Série & $3^{\text {a }}$ Série & $4^{\text {a }}$ Série & TOTAL $1^{\underline{a}}$ a $4^{\underline{a}}$ \\
\hline Anatomia & 279,0 & 158,0 & 265,0 & 54,0 & 756,0 \\
\hline Biologia Geral & 213,5 & 109,0 & 0 & 2,0 & 324,5 \\
\hline Ciências Fisiológicas & 558,0 & 363,0 & 36,0 & 0 & 957,0 \\
\hline Ciências Patológicas & 391,0 & 297,0 & 95,0 & 72,0 & 855,0 \\
\hline Histologia & 302,0 & 180,0 & 34,0 & 38,0 & 554,0 \\
\hline Microbiologia & 205,0 & 86,0 & 110,0 & 16,0 & 417,0 \\
\hline Psicologia e Psicanálise & 0 & 0 & 0 & 0 & 0 \\
\hline CENTRO DE CIÊNCIAS BIOLÓGICAS & $1.948,5$ & $1.193,0$ & 540,0 & 182,0 & $3.863,5$ \\
\hline Bioquímica e Biotecnologia & 116,5 & 0 & 0 & 24,0 & 140,5 \\
\hline Estatística & 4,0 & 0 & 0 & 0 & 4,0 \\
\hline CENTRO DE CIÊNCIAS EXATAS & 120,5 & 0 & 0 & 24,0 & 144,5 \\
\hline Ciências Sociais & 102,0 & 0 & 0 & 0 & 102,0 \\
\hline Filosofia & 98,0 & 3,0 & 0 & 0 & 101,0 \\
\hline CENTRO DE LETRAS E CIÊNCIAS HUMANAS & 200,0 & 3,0 & 0 & 0 & 203,0 \\
\hline Clínica Cirúrgica & 17,0 & 584,0 & $1.220,5$ & $1.405,0$ & $3.226,5$ \\
\hline Clínica Médica & 565,5 & 982,5 & $2.306,5$ & $2.264,0$ & $6.118,5$ \\
\hline Enfermagem & 260,0 & 154,0 & 0 & 0 & 414,0 \\
\hline Fisioterapia & 0 & 3,0 & 2,0 & 0 & 5,0 \\
\hline Ginecologia e Obstetrícia & 66,0 & 273,0 & 226,0 & 681,0 & $1.246,0$ \\
\hline Patologia, Análises Clínicas e Toxicológicas & 136,0 & 311,0 & 413,0 & 181,0 & $1.041,0$ \\
\hline Pediatria e Cirurgia Pediátrica & 253,0 & 321,0 & 357,0 & 335,0 & $1.226,0$ \\
\hline Saúde Coletiva & 550,0 & 763,0 & 31,0 & 57,0 & $1.401,0$ \\
\hline CENTRO DE CIÊNCIAS DA SAÚDE & $1.847,5$ & $3.391,5$ & $4.556,0$ & $4.923,0$ & $14.718,0$ \\
\hline Construção Civil & 0 & 3,0 & 0 & 0 & 3,0 \\
\hline CENTRO DE TECNOLOGIA E URBANISMOS & 0 & 3,0 & 0 & 0 & 3,0 \\
\hline TOTAL GERAL & $4.116,5$ & $4.590,5$ & $5.096,0$ & $5.129,0$ & $18.932,0$ \\
\hline
\end{tabular}


Verificou-se uma diminuição progressiva das horas contato docente, da primeira para a quarta série, do Centro de Ciências Biológicas, onde estão os Departamentos das Ciências Básicas, e um aumento das horas contato docente do Centro de Ciências da Saúde.

Além dos docentes vinculados à UEL, profissionais convidados/voluntários participaram das atividades acadêmicas do curso de Medicina. Foi levantado o total de $4.854,5$ horas contato dedicadas por esses profissionais, assim distribuídas: 31,0 horas na primeira série; $1.016,0$ horas na segunda; $2.183,5$ horas na terceira; e 1.624,0 horas na quarta série.

Na quinta e sexta séries do curso de Medicina, foram levantadas 33.352,0 horas contato, sendo $14.694,0$ na quinta série e $18.658,0$ na sexta série. Essa carga horária foi desempenhada por professores exclusivamente do Centro de Ciências da Saúde e dos Departamentos de Clínica Médica (11.354,0 horas), Clínica Cirúrgica (10.450,0 horas), Ginecologia e Obstetrícia (4.640,0 horas), Patologia, Análises Clínicas e Toxicológicas (68,0 horas) e Pediatria e Cirurgia Pediátrica (6.840,0 horas).

Além das atividades didáticas, os docentes realizam atividades de gerenciamento do curso. As funções docentes nas atividades de coordenação do curso de Medicina são: coordenador e vice-coordenador do Colegiado do curso; coordenador e vice-coordenador do internato; coordenador de série; coordenador e membro da Comissão de Avaliação; coordenador e mem- bro da Comissão de Capacitação; coordenador e membro da Comissão de Apoio Docente e Discente; coordenador e membro da Comissão de Acompanhamento Curricular; planejador e coordenador de módulo temático interdisciplinar; coordenador de módulo PIN; coordenador de habilidades; coordenador de módulo de atualização; e preceptor de estágio do internato.

A carga horária docente anual envolvida no gerenciamento do curso em 2005 foi de 13.137,1 horas, assim distribuídas: 1.931,6 horas destinadas à primeira série; $1.908,7$ horas à segunda; $1.880,7$ horas à terceira; $1.860,7$ horas à quarta; $2.062,7$ horas à quinta; e 3.492,7 horas à sexta série.

A maior carga horária foi utilizada na preceptoria do internato e na coordenação e planejamento dos módulos temáticos interdisciplinares. À preceptoria do internato foram dedicadas 4.290 (35,10\%) horas; à coordenação dos módulos, 3.559 (22,25\%) horas; ao planejamento dos módulos, 1.200 (9,82\%) horas; à coordenação do colegiado, 1.812 horas; à coordenação do internato, 624 horas; e 1.612 horas às comissões.

Dezessete departamentos da UEL estiveram envolvidos nas atividades de gerenciamento do curso de Medicina. As maiores cargas horárias dedicadas a essas atividades foram do Departamento de Clínica Médica, Clínica Cirúrgica e Saúde Coletiva, com 4.663, 2.867,3 e 1.537,4 horas, respectivamente. A distribuição detalhada se encontra na Tabela 2.

\section{TABELA 2}

Distribuição da carga horária anual docente envolvida no gerenciamento do curso de Graduação em Medicina da UEL segundo os Departamentos. Londrina, 2005

\begin{tabular}{|c|c|c|c|c|c|c|c|}
\hline DEPARTAMENTOS/CENTROS & 1ª Série & 2ª Série & 3a Série & $4^{a}$ Série & 5ª Série & 6⿳a Série & TOTAL \\
\hline Anatomia & 21,7 & 21,4 & 30,8 & 3,8 & 0 & 0 & 77,7 \\
\hline Biologia Geral & 178,8 & 42,3 & 26,0 & 36,0 & 26,0 & 26,0 & 335,0 \\
\hline Ciências Fisiológicas & 281,9 & 104,1 & 27,0 & 8,7 & 8,7 & 8,7 & 439,1 \\
\hline Ciências Patológicas & 25,0 & 21,8 & 9,1 & 0 & 0 & 0 & 56,0 \\
\hline Histologia & 56,2 & 40,3 & 26,0 & 26,0 & 26,0 & 26,0 & 200,5 \\
\hline Microbiologia & 238,9 & 18,8 & 17,5 & 23,7 & 8,7 & 8,7 & 316,1 \\
\hline Psicologia e Psicanálise & 17,3 & 17,3 & 17,3 & 17,3 & 17,3 & 17,3 & 104,0 \\
\hline $\begin{array}{l}\text { CENTRO DE CIÊNCIAS } \\
\text { BIOLÓGICAS }\end{array}$ & 819,8 & 266,1 & 153,7 & 115,5 & 87,0 & 87,0 & $1.528,4$ \\
\hline
\end{tabular}


TABELA 2

Distribuição da carga horária anual docente envolvida no gerenciamento do curso de Graduação em Medicina da UEL segundo os Departamentos. Londrina, 2005

\begin{tabular}{|c|c|c|c|c|c|c|c|}
\hline DEPARTAMENTOS/CENTROS & 1aㅗérie & 2ª Série & 3ª Série & $4^{a}$ Série & 5ª Série & 6ª Série & TOTAL \\
\hline Bioquímica e Biotecnologia & 162,1 & 26,0 & 31,0 & 33,1 & 26,0 & 26,0 & 304,2 \\
\hline Estatística & 5,6 & 0 & 0 & 0 & 0 & 0 & 5,6 \\
\hline CENTRO DE CIÊNCIAS EXATAS & 167,7 & 26,0 & 31,0 & 33,1 & 26,0 & 26,0 & 309,8 \\
\hline Ciências Sociais & 5,6 & 0 & 0 & 0 & 0 & 0 & 5,6 \\
\hline Filosofia & 5,6 & 0 & 0 & 0 & 0 & 0 & 5,6 \\
\hline $\begin{array}{l}\text { CENTRO DE LETRAS E CIÊNCIAS } \\
\text { HUMANAS }\end{array}$ & 11,1 & 0 & 0 & 0 & 0 & 0 & 11,1 \\
\hline Clínica Cirúrgica & 258,2 & 276,0 & 283,4 & 329,3 & 242,7 & $1.477,7$ & $2.867,3$ \\
\hline Clínica Médica & 218,2 & 623,6 & $1.007,6$ & 868,0 & 485,3 & $1.460,3$ & $4.663,0$ \\
\hline Ginecologia e Obstetrícia & 5,6 & 7,1 & 15,0 & 142,1 & 390,0 & 0 & 559,8 \\
\hline $\begin{array}{l}\text { Patologia, Análises Clínicas e } \\
\text { Toxicológicas }\end{array}$ & 3,6 & 105,7 & 60,2 & 37,2 & 130,0 & 130,0 & 466,6 \\
\hline Pediatria e Cirurgia Pediátrica & 109,2 & 276,7 & 113,2 & 113,8 & 485,3 & 95,3 & $1.193,6$ \\
\hline Saúde Coletiva & 338,3 & 327,4 & 216,7 & 221,7 & 216,7 & 216,7 & $1.537,4$ \\
\hline CENTRO DE CIÊNCIAS DA SAÚDE & 933,1 & $1.616,6$ & $1.696,0$ & $1.712,0$ & $1.950,0$ & $3.379,9$ & $11.287,7$ \\
\hline TOTAL GERAL & $1.931,6$ & $1.908,7$ & $1.880,7$ & $1.860,7$ & $2.062,7$ & $3.492,7$ & $13.137,0$ \\
\hline
\end{tabular}


As atividades da primeira à quarta série implicaram um custo geral de $\mathrm{R} \$ 855.586,74$ - R\$ 215.249,15 na primeira série; $\mathrm{R} \$$ 220.703,70 na segunda; $\mathrm{R}$ \$ 193.321,91 na terceira; e R \$226.311,97 na quarta série. O custo aluno anual foi de $\mathrm{R} \$ 2.690,61$ na primeira série; $\mathrm{R}$ \$ 2.691,51 na segunda; $\mathrm{R}$ 1.993,01 na terceira; e $\mathrm{R} \$$ 2.726,65 na quarta série (Tabela 3).

Verificou-se que os custos do Centro de Ciências Biológicas diminuíram progressivamente da primeira para a quarta série, com aumento dos custos do Centro de Ciências da Saúde, como já descrito em relação às horas contato docente.

As atividades da quinta e sexta séries implicaram um custo geral de $\mathrm{R} \$ 1.629 .025,93$, sendo $\mathrm{R}$ \$ 1.085.097,88 da quinta série e
R\$ 543.928,05 da sexta série (Tabela 4). O custo aluno anual da quinta série foi de $\mathrm{R} \$ 13.735,42$ e da sexta série foi de $\mathrm{R} \$ 6.715,16$.

As atividades de gerenciamento do curso representaram um custo geral de $\mathrm{R} \$ 586.642,21$ e custo aluno anual de $\mathrm{R} \$ 1.168,61$. O custo do Centro de Ciências da Saúde foi estimado em R\$ $486.501,68$, representando $82,9 \%$ do custo administrativo. O custo do Centro de Ciências Biológicas foi de R\$78.668,65, do Centro de Ciências Exatas foi de $\mathrm{R} \$ 20.983,98$, e do Centro de Letras e Ciências Humanas foi de R $\$ 487,90$.

TABELA 3

Distribuição do custo da primeira a quarta séries do curso de graduação em Medicina da UEL por Departamentos. Londrina, 2005

\begin{tabular}{|c|c|c|c|c|c|}
\hline DEPARTAMENTOS/CENTROS & $1^{\text {a }}$ Série & 2ª Série & 3a Série & $4^{\mathrm{a}}$ Série & TOTAL1 ${ }^{\mathrm{a}}$ a $4^{\mathrm{a}}$ \\
\hline Anatomia & $12.284,05$ & $6.956,56$ & $11.667,65$ & $2.377,56$ & $33.285,81$ \\
\hline Biologia Geral & $11.006,57$ & $5.619,28$ & 0,00 & 103,11 & $16.728,96$ \\
\hline Ciências Fisiológicas & $33.041,83$ & $17.586,78$ & $2.131,73$ & 0,00 & $52.760,34$ \\
\hline Ciências Patológicas & $21.229,07$ & $16.125,40$ & $5.157,96$ & $3.909,19$ & $46.421,62$ \\
\hline Histologia & $5.761,84$ & $3.434,21$ & 648,68 & 725,00 & $10.569,74$ \\
\hline Microbiologia & $13.349,75$ & $5.600,38$ & $7.163,28$ & $1.041,93$ & $27.155,35$ \\
\hline Psicologia e Psicanálise & 0,00 & 0,00 & 0,00 & 0,00 & 0,00 \\
\hline CENTRO DE CIÊNCIAS BIOLÓGICAS & $96.673,11$ & $55.322,62$ & $26.769,30$ & $8.156,78$ & $186.921,81$ \\
\hline CUSTO ALUNO/ANO/CENTRO & $1.208,41$ & 691,53 & 334,62 & 101,96 & $2.336,52$ \\
\hline Bioquímica e Biotecnologia & $8.034,81$ & 0,00 & 0,00 & $1.655,24$ & $9.690,06$ \\
\hline Estatística & $6.403,62$ & 0,00 & 0,00 & 0,00 & $6.403,62$ \\
\hline CENTRO DE CIÊNCIAS EXATAS & $14.438,43$ & 0,00 & 0,00 & $1.655,24$ & $16.093,68$ \\
\hline CUSTO ALUNO/ANO/CENTRO & 180,48 & 0,00 & 0,00 & 20,69 & 201,17 \\
\hline
\end{tabular}


TABELA 3

Distribuição do custo da primeira a quarta séries do curso de graduação em Medicina da UEL por Departamentos. Londrina, 2005

\begin{tabular}{|c|c|c|c|c|c|}
\hline DEPARTAMENTOS/CENTROS & 1ํㅗㄹie & 2ª́ Série & 3a Série & $4^{a}$ Série & TOTAL1 $1^{\mathrm{a}}$ a $4^{\mathrm{a}}$ \\
\hline Ciências Sociais & $4.602,95$ & 0,00 & 0,00 & 0,00 & $4.602,95$ \\
\hline Filosofia & $4.192,46$ & 128,34 & 0,00 & 0,00 & $4.320,80$ \\
\hline $\begin{array}{l}\text { CENTRO DE LETRAS E CIÊNCIAS } \\
\text { HUMANAS }\end{array}$ & $8.795,41$ & 128,34 & 0,00 & 0,00 & $8.923,75$ \\
\hline CUSTO ALUNO/ANO/CENTRO & 109,94 & 1,60 & 0,00 & 0,00 & 111,55 \\
\hline Clínica Cirúrgica & 476,22 & $16.359,40$ & $34.189,47$ & $39.357,80$ & $90.382,89$ \\
\hline Clínica Médica & $17.220,26$ & $29.918,48$ & $70.236,11$ & $68.941,93$ & $186.316,78$ \\
\hline Enfermagem & $7.902,40$ & $4.680,65$ & 0,00 & 0,00 & $12.583,06$ \\
\hline Fisioterapia & $2.984,81$ & 83,91 & 55,94 & 0,00 & $3.124,66$ \\
\hline Ginecologia e Obstetrícia & $7.464,75$ & $30.876,93$ & $25.561,12$ & $77.022,66$ & $140,925,46$ \\
\hline Patologia, Análises Clínicas e Toxicológicas & $3.326,13$ & $7.606,09$ & $10.100,699$ & $4.426,69$ & $25.459,60$ \\
\hline Pediatria e Cirurgia Pediátrica & $17.165,93$ & $21.779,70$ & $24.222,28$ & $22.729,59$ & $85.897,51$ \\
\hline Saúde Coletiva & $38.801,70$ & $53.828,54$ & $2.187,00$ & $4.021,27$ & $98.838,51$ \\
\hline CENTRO DE CIÊNCIAS DA SAÚDE & $95.342,20$ & $165.133,70$ & $166.552,61$ & $216.499,95$ & $643.528,46$ \\
\hline CUSTO ALUNO/ANO/CENTRO & $1.191,78$ & $2.064,17$ & $2.081,91$ & $2.706,25$ & $8.044,11$ \\
\hline Construção Civil & 0,00 & 119,05 & 0,00 & 0,00 & 119,05 \\
\hline $\begin{array}{l}\text { CENTRO DE TECNOLOGIA E } \\
\text { URBANISMOS }\end{array}$ & 0,00 & 119,05 & 0,00 & 0,00 & 119,05 \\
\hline CUSTO ALUNO/ANO/CENTRO & & & & & \\
\hline TOTAL GERAL & $215.249,15$ & $220.703,70$ & $193.321,91$ & $226.311,97$ & $855.586,74$ \\
\hline Número de alunos matriculados na série & 80 & 82 & 97 & 83 & 342 \\
\hline CUSTO ANO ALUNO DA SÉRIE & $2.690,61$ & $2.690,51$ & $1.993,01$ & $2.726,65$ & $2.501,72$ \\
\hline
\end{tabular}


TABELA 4

Distribuição do custo da quinta e sexta séries do curso de graduação em Medicina da UEL por Departamentos. Londrina, 2005

\begin{tabular}{lccc}
\hline DEPARTAMENTOS/CENTRO & $5^{\mathrm{a}}$ Série & $6^{\text {a }}$ Série & Total $5^{\mathrm{a}}$ e $6^{\mathrm{a}}$ \\
\hline & & & $292.732,42$ \\
Clinica Cirúrgica & $19.048,62$ & $273.683,80$ & $345.744,98$ \\
Clinica Médica & $77.163,80$ & $268.581,18$ & $524.794,66$ \\
Ginecologia e Obstetrícia & $524.794,66$ & 0,00 & $1.663,07$ \\
Patologia, Análises Clínicas e Toxicológicas & 0,00 & $1.663,07$ & $464.090,81$ \\
Pediatria e Cirurgia Pediátrica & $464.090,81$ & 0,00 & $1.629 .025,93$ \\
CENTRO DE CIÊNCIAS DA SAÚDE & $1.085 .097,88$ & $543.928,05$ & $1.629 .025,93$ \\
TOTAL GERAL & $1.085 .097,88$ & $543.928,05$ & 160 \\
Número de alunos matriculados na série & 79 & 81 & $10.181,41$ \\
CUSTO ANO ALUNO DA SÉRIE & $13.735,42$ & $6.715,16$ & \\
\hline
\end{tabular}

O custo total do curso de graduação em Medicina, considerando as horas contato dos professores nas atividades acadêmicas da primeira a sexta séries e as atividades de gerenciamento do curso, foi estimado em R\$3.071.254,89. Dividindo-se esse total pelo número de alunos matriculados no ano, obteve-se o custo anual do aluno: $\mathrm{R} \$ 6.118,04$ (Tabela 5).

Acrescentou-se também o custo estimado do hospital universitário no curso de graduação em Medicina, e o custo do curso foi de $\mathrm{R} \$ 15.643 .660,89$, o que representou um custo aluno anual de $\mathrm{R} \$ 31.162,67$ e custo aluno mensal de $\mathrm{R} \$ 2.596,89$. Esse valor, convertido em dólar americano pela média anual praticada no mercado financeiro do ano de 2005, representou US\$ 10,633.86 de custo aluno anual e US\$ 886.15 de custo aluno mensal.

TABELA 5

Custo do curso de graduação em Medicina da UEL em reais e dólares por Departamentos. Londrina, 2005

\begin{tabular}{|c|c|c|c|c|c|}
\hline \multirow{2}{*}{$\begin{array}{l}\text { DEPARTAMENTOS } \\
\text { ENVOLVIDOS }\end{array}$} & \multirow{2}{*}{$\begin{array}{l}\text { CUSTO } \\
\text { DEPT }^{\circ}\end{array}$} & \multicolumn{2}{|c|}{ CUSTO EM R\$ } & \multicolumn{2}{|c|}{ CUSTO EM US\$ } \\
\hline & & ANUAL & MENSAL & ANUAL & MENSAL \\
\hline Anatomia & $36.706,64$ & 73,12 & 6,09 & 24.95 & 2.08 \\
\hline Biologia Geral & $34.001,14$ & 67,73 & 5,64 & 23.11 & 1.93 \\
\hline Ciências Fisiológicas & $78.759,56$ & 156,89 & 13,07 & 53.54 & 4.46 \\
\hline Ciências Patológicas & $49.459,50$ & 98,52 & 8,21 & 33.62 & 2.80 \\
\hline Histologia & $14.395,52$ & 28,68 & 2,39 & 9.79 & 0.82 \\
\hline Microbiologia & $47.743,02$ & 95,11 & 7,93 & 32.45 & 2.70 \\
\hline Psicologia e Psicanálise & $4.525,09$ & 9,01 & 0,75 & 3.08 & 0.26 \\
\hline
\end{tabular}


TABELA 5

Custo do curso de graduação em Medicina da UEL em reais e dólares por Departamentos. Londrina, 2005

\begin{tabular}{|c|c|c|c|c|c|}
\hline \multirow{2}{*}{$\begin{array}{l}\text { DEPARTAMENTOS } \\
\text { ENVOLVIDOS }\end{array}$} & \multirow{2}{*}{$\begin{array}{l}\text { CUSTO } \\
\text { DEPT }^{\circ}\end{array}$} & \multicolumn{2}{|c|}{ CUSTO EM R\$ } & \multicolumn{2}{|c|}{ CUSTO EM US\$ } \\
\hline & & ANUAL & MENSAL & ANUAL & MENSAL \\
\hline Bioquímica e Biotecnologia & $30.674,04$ & 61,10 & 5,09 & 20.85 & 1.74 \\
\hline Estatística & $6.403,62$ & 12,76 & 1,06 & 4.35 & 0.36 \\
\hline Ciências Sociais & $4.853,41$ & 9,67 & 0,81 & 3.30 & 0.27 \\
\hline Filosofia & $4.558,24$ & 9,08 & 0,76 & 3.10 & 0.26 \\
\hline Clinica Cirúrgica & $463.436,32$ & 923,18 & 76,93 & 315.02 & 26.25 \\
\hline Clinica Médica & $674.058,74$ & $1.342,75$ & 111,90 & 458.19 & 38.18 \\
\hline Enfermagem & $12.583,06$ & 25,07 & 2,09 & 8.55 & 0.71 \\
\hline Fisioterapia & $3.124,66$ & 6,22 & 0,52 & 2.12 & 0.18 \\
\hline Ginecologia e Obstetrícia & $729.041,20$ & $1.452,27$ & 121,02 & 495.57 & 41.30 \\
\hline Patologia, Análises Clínicas e Tox. & $38.534,95$ & 76,76 & 6,40 & 26.19 & 2.18 \\
\hline Pediatria e Cirurgia Pediátrica & $630.972,91$ & $1.256,92$ & 104,74 & 428.91 & 35.74 \\
\hline Saúde Coletiva & $207.304,25$ & 412,96 & 34,41 & 140.92 & 11.74 \\
\hline Construção Civil & 119,05 & 0,24 & 0,02 & 0.08 & 0.01 \\
\hline TOTAL ACADÊMICO & $3.071 .254,89$ & $6.118,04$ & 509,84 & $2,087.70$ & 173.98 \\
\hline Hospital Universitário & $12.572 .406,00$ & $25.044,63$ & $2.087,05$ & $8,546.16$ & 712.18 \\
\hline TOTAL GERAL & $15.643 .660,89$ & $31.162,67$ & $2.596,89$ & $10,633.86$ & 886.15 \\
\hline Número de alunos matriculados & 502 & & & & \\
\hline
\end{tabular}




\section{DISCUSSÃO}

A literatura sobre custos na área de educação brasileira ainda é bastante reduzida. Alguns trabalhos produzidos por pesquisadores de universidades públicas têm tratado do assunto, sem, entretanto, conseguir institucionalizar uma metodologia que atenda às peculiaridades das instituições de ensino superior e às especificidades de cada curso.

Convém destacar que o estudo investigou exclusivamente as horas docentes em contato com o estudante e as horas destinadas ao gerenciamento do curso. Além destas, os professores desenvolvem atividades de pesquisa, extensão, pós-graduação (residência médica), administração de outras instâncias (direção de Centro, Departamentos, comissões do Centro, entre outras) e, principalmente, atividades de atenção à saúde.

Em 2005, para desenvolver o curso de graduação em Medicina da UEL, no qual estavam matriculados 502 estudantes, foram necessários 284 professores (equivalentes a 222,2 professores em tempo integral).

O custo estimado para as atividades acadêmicas da primeira à sexta série e as atividades de gerenciamento foi de $\mathrm{R} \$$ 3.071.254,89. A apuração do custo estimado do hospital universitário quadruplicou o valor encontrado, passando para R\$ 15.643.660,89. O custo aluno anual foi estimado em $\mathrm{R} \$ 31.162,67$, o equivalente a US\$10,633.86.

A análise de custos nas instituições de ensino superior brasileiras revela que o curso de Medicina é o de maior custo. Silva et al..$^{29}$ realizaram a apuração de custo de uma instituição federal e verificaram que os maiores custos foram das unidades de Medicina, Ciências da Saúde e Geociências, e os menores custos foram das unidades de Letras, Direito e Ciências Sociais. O custo aluno anual na Faculdade de Medicina foi de R\$ 19.074,00 e R\$ 16.115,00 nos anos de 2002 e 2003, respectivamente. A redução no custo apurado em 2003 deveu-se principalmente ao aumento do número de alunos.

A aplicação do Sistema de Apuração de Custos (SAC) na Universidade Federal do Ceará, com dados do exercício de 1997, apurou o custo médio por aluno de Medicina de $\mathrm{R} \$ 7.551,08^{30}$. OSAC foi desenvolvido pela Secretaria de Educação Superior do Ministério da Educação (SESu/MEC) para avaliação gerencial das instituições federais de ensino superior. O sistema foi desenvolvido e distribuído gratuitamente a todas as instituições de ensino interessadas em sua utilização. Peter et al. ${ }^{30}$ referem-se ao desconhecimento das Ifes em relação à existência e operacionalização do sistema, e alguns problemas já foram detectados. Os autores referem que, apesar de os resultados apontarem a Faculdade de Medicina com o maior custo-aluno na instituição pesquisada, este custo está aquém dos levantados nos Estados Unidos.
Outras experiências de apuração de custo mostram um resultado elevado de custo por aluno, se comparado ao encontrado na presente pesquisa.

Goodwin et al. ${ }^{27}$ apuraram, no curso de Medicina da Virginia Commonwealth University, no ano de 1994-95, um custo total de US\$ 47,174,437, sendo US\$ 35,068,716 de custos diretos. O custo anual por aluno foi de US\$ 69,992 (dólares 1995). Os autores verificaram a participação de 201 docentes em regime integral para 674 estudantes matriculados. Do total de professores, 13 eram das Ciências Básicas e 188 das Clínicas. Foram levantadas 89.223 horas contato.

Um estudo na Universidade do Texas - Houston Medical School, realizado por Rein et al. ${ }^{10}$, concluiu que para o ensino médico de 556 estudantes participaram 223 professores em tempo integral, sendo 7 das Ciências Básicas e 216 das Ciências Clínicas e residentes. O estudo apresentou somente o custo aluno anual de US\$ 89,258 (dólares 1995).

Franzini et al. ${ }^{11}$ referem que, para ensinar 800 alunos, foram requeridos 201 docentes em tempo integral, e 258 residentes deram suporte ao ensino. O custo aluno anual estimado foi de US \$43,993.

A análise de custo de Ginzberg et al., apud Jones e Korn ${ }^{31}$, mostrou um custo mais baixo e próximo do encontrado no presente estudo. Os autores apuraram o custo de US\$ 6.7 milhões para 600 estudantes de Medicina, o equivalente a US\$11,375 (dólares 1992). Jones e Korn ${ }^{31}$ atribuíram o menor custo ao fato de os autores terem considerado na análise somente as horas contato direto com o aluno, não tendo incluído o tempo de preparo de aula, o desenvolvimento profissional, as atividades administrativas e a participação dos residentes no ensino.

Em relação ao alto custo do hospital universitário, Silva ${ }^{32}$ cita que diversos fatores, decorrentes de atividades de ensino, podem elevar os custos dos hospitais universitários. Estudantes podem utilizar mais recursos mesmo quando não executam nenhum procedimento, como, por exemplo, luvas e máscaras, para observar a manipulação de um medicamento quimioterápico. Podem, ainda, dosar um medicamento incorretamente, necessitando do preparo de outra dose. Uma cirurgia pode durar um tempo maior em virtude das atividades de ensino e, conseqüentemente, aumentar os gastos com depreciação, pessoal, materiais e equipamentos e medicamentos.

Médici ${ }^{33}$ refere que os HU são instituições caras, por concentrarem atendimentos de alta complexidade, além de atividades que mesclam procedimentos didáticos. É possível que atividades que envolvam ensino incluam certa dose de ineficiência técnica implícita ao processo didático. Segundo o autor, os hospitais universitários têm características que fazem com que seus cus- 
tos sejam ainda mais elevados que os dos hospitais não-universitários de alta tecnologia, tais como incorporação das atividades docentes-assistenciais e prestação de serviços básicos de saúde, quando sua estrutura é constituída para oferecer atividades de alta tecnologia.

Sobre à variação de custos encontrada nos estudos, Jones e $K{ }^{31}$ referem que ela pode estar mais relacionada às definições de custos associadas à realização ou não de pesquisas e assistência a pacientes do que às diferenças entre as instituições.

Morgan ${ }^{18}$ cita algumas variáveis que podem presumir o comportamento dos custos na graduação: área física, dedicação do corpo docente ao ensino, custo dos bens móveis, número de professores doutores, número de professores mestres, número de professores especialistas, número de professores graduados, número de professores em dedicação exclusiva e/ou 40 horas, número de professores de 20 horas ou outro regime de trabalho, número de funcionários e número de alunos da graduação.

Soares e Cericato ${ }^{34}$ mostram a dificuldade que existe na comparação entre os valores dos custos dos alunos entre as universidades nacionais e entre universidades de diferentes países. As universidades brasileiras apresentam diferentes metodologias de cálculo e dificuldades de alocação de custo, fatores que dificultam a correta mensuração do custo aluno.

Já as comparações internacionais de custo aluno são incongruentes devido a fatores como políticas de ingresso nas universidades, situação legal das instituições de ensino superior, quadro de pessoal, tradição administrativa e leis do país ${ }^{35}$.

O chamado Cost Construction Model usado nesta pesquisa é um instrumento poderoso e permite a análise dos chamados custos instrucionais. Assim, ele "descontamina" a análise de custos totais de variáveis metodológicas e institucionais que são responsáveis pela variabilidade dos resultados obtidos na literatura.

A apuração do custo do curso de Medicina da UEL mostrou a importância de efetuar essa análise considerando as características de um curso específico, levando-se em conta seu desenho curricular, uma vez que a apuração de forma global para a instituição de ensino oferece poucas informações que sirvam para análises comparativas.

\section{REFERÊNCIAS}

1. Velloso J. Custos reais e custos contábeis da universidade pública. In: Velloso J. Universidade pública: política, desempenho, perspectivas. Campinas: Papirus;1991.

2. Horngren CT, Foster G, Datar SM. Contabilidade de custos. 9.ed. Rio de Janeiro: LTC; 2000.
3. Maher M. Contabilidade de Custos - criando valor para a administração. São Paulo: Atlas; 2001.

4. Lima JG. Custos:cálculos, sistemas e análises. 2. ed. São Paulo: Atlas; 1980.

5. Megliorini E. Custos. São Paulo: Makron Books; 2001.

6. Calil VLLO. Estruturação de um sistema de integração orçamentária, financeira e de custos parara as Universidades Estaduais do Paraná. [tese] Florianópolis: Universidade Federal de Santa Catarina; 2005.

7. Yoshitake M. Teoria do controle de gestão. BRADEM. Instituto Brasileiro de Doutores e Mestres em Ciências Contábeis: Salvador; 2002.

8. Carpintéro JN, Bacic MJ. Metodologia de Cálculo do Custo Aluno no Ensino Fundamental. Campinas: Instituto de Economia da Universidade de Campinas; 1999.

9. Cooper JAD. Cost estimates of the undergraduate medical education program - the Association's findings and those of the Medicine (memorandum). Washington, DC: Association of American Medical Colleges; 1974.

10. Rein MF, Randolph WJ, Short JG, Coolidge KG, Coates ML, Carey RM, et al. Defining the cost of educating undergraduate medical students at the University of Virginia. Acad Med. 1997;72:218-227.

11. Franzini L, Low MD, Proll MA. Using a cost-construction model to assess the cost of educating Undergraduate medical students at the University of Texas-Houston Medical School. Acad Med. 1997;72(3):228-237.

12. Brasil. Tribunal de Contas da União. Relatório Consolidado de Auditoria Operacional. Brasília: Tribunal de Contas da União; 2002. Decisão Plenária n. 408.

13. Brasil. Ministério da Educação. Secretaria de Educação Superior. Dados e Indicadores das Instituições Federais de Ensino Superior 2000. Brasília: MEC; 2002.

14. Brasil. Ministério da Educação. Instituto Nacional de Estudos e Pesquisas Educacionais Anísio Teixeira (INEP). Censo da Educação Superior: Brasília: MEC; 2003. Disponível em: http:/ / www.inep.gov.br. [Acesso em: 20 de Abril de 2007].

15. Universidade de Brasília. Assessoria de Planejamento e Controle. Modelo para estimar custos e alocar recursos nas universidades. Brasília: UNB; 1973.

16. Universidade de Brasília. Estudo dos resultados da aplicação do modelo de apropriação de custo da UFBA. Brasília: UNB; 1974. 
17. Brasil. Ministério da Educação e do Desporto. Secretaria de Educação Superior. Sistema de Apuração de Custos das Instituições Federais de Ensino Superior. Brasília;1994.

18. Morgan BF. A Determinação do Custo do Ensino Na Educação Superior: o caso da Universidade de Brasília. [dissertação] Brasília: Universidade de Brasília; 2004.

19. Mennin SP; Martinez-Burrola N. The cost of problem-based vs. traditional medical education. Med Educ. 1986;20(3):187-194.

20. Nieuwenhuijzen Kruseman AC, Kolle LFJ, Scherpbier AJJ. Problem-based learning at Maastricht-an assessment of cost and outcome. J. Education for Health. 1997; 10(2):179-187.

21. Vimolket T, Kamol-Ratanakul P, Indaratna K. Cost of Producing a Medical Doctor at Chulalongkorn University. J Med Assoc Thai 2003; 86 (1): 82-92.

22. Brown WA, Gamber C. Cost containment in higher education: issues and recommendations. ASHE-ERIC Higher Education Report 2002; 28(5):161.

23. Universidade Estadual de Londrina. Colegiado do Curso de Medicina. Projeto Político-Pedagógico do curso de Medicina. Londrina; 2005.

24. Beuren IM. Como elaborar trabalhos monográficos em Contabilidade: teoria e prática. São Paulo: Atlas; 2003.

25. Otley DT, Berry AJ. Case study research in management accounting and control Management Accounting Research 1994; 5:45-65.

26. Valberg LS, Gonyea MA, Sinclair DG, Wade J. Planning the future Academic Medical Centre. CMAJ. 1994;151(11): 1581-1587.

27. Goodwin MC, Gleason WM, Kontos HA. A Pilot study on the cost of educating undergraduate medical students at Virginia Commonwealth University. Acad Med. 1997;72(3):211-217.
28. Universidade Estadual de Londrina. Pró-Reitoria de Planejamento. Custos. Disponível em; http:/ / www.uel.br/proplan. [Acesso em: 20 de Abril de 2007].

29. Silva CAT, Morgan BF, Costa OS. Apuração do custo por aluno: aplicação a uma instituição federal de ensino superior. XXVIII Encontro da ANPAD; 25 - 29 set.2004;Curitiba: ANPAD ; 2004.

30. Peter MGA, Pessoa MNM, Pinho RCS, Peter FA. Sistema de apuração de custos das Universidades Federais Brasileiras: uma análise crítica. Anais do VIII Congresso del Instituto Nacional de Costos. Punta Del Este,Uruguai; 2003.

31. Jones RF, Korn D. On the cost of educating a medical student. Acad Med. 1997;72(3):200-210.

32. Silva CAT. Relatório sobre custo/aluno 2004. Disponível em: http://www.spl.unb.br. [Acesso em: 20 de Abril de 2007].

33. Medici AC. Hospitais Universitários: Passado, Presente e Futuro. Rev Ass Med Brasil 2001;47(2):149-156.

34. Soares TC, Cericato D. Custo por aluno: algo de fácil comparação? V Colóquio Internacional sobre Gestión Universitária em América del Sur. dez. 2005; Mar del Plata; 2005.

35. Sheehan J. Modelos para la asignación de los fondos públicos entre las universidades. Ministerio de Educación de Argentina. Nuevas direcciones em el financiamiento de la educación superior; 1996. Disponível em http://www.me.gov.ar. [Acesso em: 22 de Agosto de 2005

\section{CONFLITOS DE INTERESSE}

Declarou não haver.

\section{ENDEREÇO PARA CORRESPONDÊNCIA}

Celita Salmaso Trelha Rua Alagoas, 1110 - apto 303 - Centro 86020-430 - Londrina - Paraná celita@dilk.com.br 